\title{
Terremoto 2010: Impacto en la incidencia de lesiones subepicárdicas. experiencia en telemedicina
}

Francesca Bello, Patricia Adriazola, Edgardo Escobar, Stefania Pavlov, Gabriel Mezzano, Denisse Lama.

ITMS Telemedicina de Chile.

\section{Resúmen}

La asociación de estrés y eventos coronarios agudos ha sido extensamente estudiada. En relación a un evento estresante existiría un aumento en la liberación de catecolaminas que incide en un incremento en la presión arterial y alteraciones de hemostasia, como sería el caso de un terremoto.

En el presente trabajo se describe la relación contemporánea observada entre el terremoto del 27 de febrero del 2010 y el diagnóstico de lesión subepicárdica, en electrocardiogramas recibidos en ITMS, Telemedicina de Chile.

Se analizaron 280.592 electrocardiogramas (ECGs) provenientes de todo el territorio nacional, que corresponden a un periodo de 12 meses de los años 2009 y 2010. Se realizó una comparación estadística entre el porcentaje de lesiones subepicárdicas registradas en el fin de semana del terremoto (27/02/2010 y 28/02/2010) versus los fines de semana de los meses estudiados. Se obtuvieron los datos correspondientes a siete pacientes que se encontraban con monitoreo ambulatorio de presión arterial (MAPA) el día 27/02.

El diagnóstico de lesión subepicárdica se hizo en 1.795 trazados, correspondientes a un $0.64 \%$ del total de ECGs recibidos. Hubo un aumento estadísticamente significativo de las lesiones subepicárdicas $(\mathrm{p}<0,05)$ en el fin de semana del terremoto, la distribución por edad se mantuvo similar al resto de los períodos analizados. Sin embargo se invirtió la distribución por género durante dicho fin de semana siendo mayor en mujeres que en hombres, como es en el resto de los períodos analizados. En los registros MAPA se documentó un aumento de la presión arterial y frecuencia cardíaca en relación al evento estresante.

Se confirmó entonces un aumento estadísticamente significativo del diagnóstico electrocardiográfico de lesiones subepicárdicas en relación al estrés producido por el terremoto del 27 de febrero de 2010.

\section{Correspondencia:}




\section{Impact of the 2010 earthquake and tsunami in Chile: a telemedicine evaluation of subepicardial lesions}

Background: The association of stress and acute coronary events has been extensively explored. An increased output of catecholamines leading to high blood pressure and alteration of hemostasis may be responsible for this effect. A severe earthquake and tsunami is a major stress.

Methods and Results. 280,592 electrocardiograms (ECG) obtained via a telemedicine system in the years 2009 and 2010 were analyzed. The frequency of subepicardial lesions recorded during the weekend in which the earthquake took place (27/2/2010 - 28/2/2010) was compared to that observed in all other weekends throughout the period. 7 subjects had a blood pressure Holter monitoring on that weekend. A subepicardial lesion was diagnosed in $0.64 \%$ of
ECGs recorded throughout the period, increasing significantly in the earthquake's weekend $(\mathrm{p}<0.05)$. The age distribution of subepicardial lesions was similar in all weekends. However, the higher proportion of males with sub epicardial lesions throughout the study period changed to a higher proportion of females in the earthquake's weekend. Significant increases in blood pressure and heart rate were observed in coincidence with the earthquake in patients undergoing blood pressure monitoring.

Conclusion: a statistically significant increase in subepicardial lesions demonstrated by telemedicine ECG recordings was associated to the stressful situation of the 2010 earthquake in Chile.

\section{Keywords: earthquake, telemedicine, ECG}

\section{Introducción}

La asociación de estrés y eventos coronarios agudos (ECA) ha sido extensamente estudiada ${ }^{1-6}$, planteándose la posibilidad que eventos estresantes crónicos y agudos estén relacionados con un aumento en el riesgo cardiovascular $^{7}$

De los eventos estresantes agudos "naturales" se ha documentado la relación entre terremoto y ECA. Se ha planteado una relación entre la intensidad del terremoto, la hora de ocurrencia, el lugar geográfico y el riesgo de $\mathrm{ECA}^{3}$.

En relación a un evento estresante existe un aumento en la liberación de catecolaminas (hiperactividad simpática) que incide en un incremento en la presión arterial (PA) y alteraciones de hemostasia, incremento de viscosidad sanguínea y de marcadores de actividad procoagulante que explicarían el riesgo aumentado de ECA en relación a un estrés mayor ${ }^{3}$.

Un ejemplo se evidencia en el terremoto de HanshinAwaji donde se observó un aumento en 3 veces la incidencia histórica de Infarto agudo del miocardio (IAM) en población cercana al epicentro, predominantemente en mujeres. En relación a este evento se documentó la asociación entre el aumento de enfermedad cardiovascular, cambios hemodinámicos y factores trombogéni$\cos ^{4}$.
En el presente trabajo se describe la relación contemporánea observada entre el terremoto del 27 de febrero del 2010 y el diagnóstico de lesión subepicárdica, en electrocardiogramas recibidos en ITMS, Telemedicina de Chile, sistema que permite estudiar este tipo de fenómenos en tiempo real.

El sistema de Telemedicina está distribuido a lo largo y ancho de todo Chile, comprendiendo tanto al sistema público de salud como al ámbito privado y ha permitido estudiar la relación del intervalo QT en diferentes patrones electrocardiográficos, la prevalencia de la morfología de Brugada y la incidencia de lesiones subepicárdicas.

\section{Método}

Se analizaron 280.592 electrocardiogramas (ECGs) obtenidos de la base de datos del sistema de Telemedicina de Chile, provenientes de todo el territorio nacional, que corresponden a un período de 12 meses:

- Enero, Febrero, Marzo del año 2009

- Enero, febrero y Marzo del año 2010

- Junio, Julio, Agosto del año 2009

- Junio, julio y Agosto del año 2010

Se revisó el porcentaje diario de lesiones subepicárdicas diagnosticadas en el ECG y para la comparación estadística entre el porcentaje de lesiones subepicárdi- 
cas registradas en el período inmediatamente posterior al terremoto (27/02/2010 y 28/02/2010) versus los fines de semana de los meses estudiados correspondientes a los años 2009 y 2010, se utilizó la prueba de $\mathrm{Chi}^{2}$.

De manera simultánea se obtuvieron los datos correspondientes en siete pacientes que se encontraban con monitoreo ambulatorio de presión arterial (MAPA), donde se pudo evaluar la variación en la frecuencia cardíaca y presión arterial en relación al terremoto, comparando el porcentaje de variación del promedio de las cuatro mediciones previas a la hora del terremoto con la medición en la hora del evento.

En forma diferida se realizó el seguimiento de los pacientes con diagnóstico de lesión subepicárdica en el fin de semana post terremoto y se obtuvieron datos respecto del estudio y la terapia que recibieron.

\section{Resultado}

De los ECGs estudiados se hizo el diagnóstico de lesión subepicárdica en 1.795 trazados, correspondientes a un $0.64 \%$ del total.

La distribución por edad se mantuvo similar en los 12 meses evaluados. El promedio de edad de presentación de lesión subepicárdica ( \pm DE) fue de $62 \pm 4,8$ años) (mujeres $67,7 \pm 14,6$ años); hombres $60 \pm 14,4$ años). Se mantuvo la diferencia en edad de presentación según género, la cual es mayor en mujeres.

En el promedio general de las lesiones subepicárdicas $70 \%$ correspondieron a hombres y un $30 \%$ a mujeres. 48

Figura 1: distribución de los porcentajes de lesiones subepicárdicas durante los fines de semana

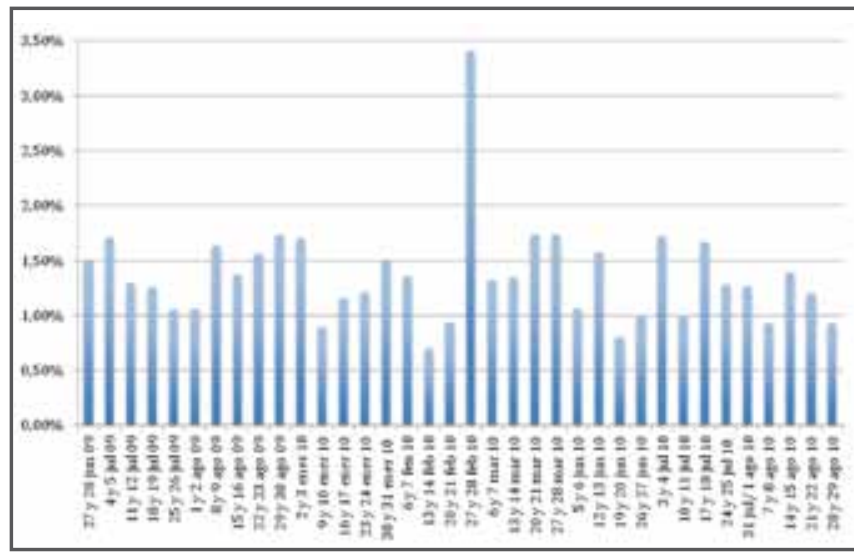

Distribución de los porcentajes promedios de lesiones subepicárdicas analizadas en los fines de semanas de los doce meses estudiados; destaca aumento significativo el fin de semana del terremoto. horas post terremoto esta relación se invierte, un $75 \%$ correspondieron a mujeres y un $25 \%$ a hombres.

En la figura 1 se observa la distribución de los porcentajes promedios de lesiones subepicárdicas analizadas en los fines de semanas de los doce meses estudiados; destaca el significativo aumento en diagnóstico de lesión subepicárdica durante fin de semana correspondiente al terremoto. Se comparó estadísticamente el porcentaje de lesiones subepicárdicas durante el fin de semana post terremoto con los fines de semana de los restantes meses analizados, demostrando que hubo un aumento estadísticamente significativo de las lesiones subepicárdicas $(\mathrm{p}<0,05)$.

$\mathrm{Al}$ analizar las comorbilidades de los 12 pacientes con ECG compatibles con lesión subepicárdica en el período posterior al terremoto destaca que un $33 \%$ no tenía comorbilidades conocidas al momento del diagnóstico, un 50\% eran hipertensos, más del $40 \%$ tenían antecedentes de enfermedad coronaria y menos del $10 \%$ tenía antecedentes de Diabetes mellitus tipo 2.

$\mathrm{Al}$ investigar el tratamiento y estudio al que fueron sometidos estos pacientes no fue posible obtener antecedentes en el 58\% de los casos (7 pacientes) porque no se contaba con registro adecuado en los sitios de origen. De estos casos se documentó el fallecimiento de un paciente meses después, secundario a IAM extenso.

De los 5 pacientes con antecedentes, 3 fueron sometidos a trombolisis al momento del diagnóstico electrocardiográfico, 2 de ellos fueron derivados al Hospital Salvador donde se realizó coronariografía en diferido; en ambos casos se documentó enfermedad aterosclerótica extensa. Dos pacientes fueron sometidos a coronariografía primaria, y de ellos, uno tenía coronariografía normal y el otro fue sometido a angioplastía primaria.

En el registro de MAPA de 7 pacientes en el mismo día a la hora de la catástrofe se observa un aumento de la frecuencia cardíaca de un $29 \%$ sobre el promedio de las cuatro mediciones previas a la hora del terremoto con un porcentaje máximo de variación de un $58,4 \%$ (paciente B) y un mínimo de $12 \%$ (pacientes E y G). En relación a presión arterial diastólica se observó un aumento promedio de un $33,2 \%$ con un máximo de $61,2 \%$ (paciente B) y un mínimo de 7,2\% (paciente E), en presión arterial sistólica un porcentaje de aumento de $24,8 \%$ con un máximo de $59,7 \%$ (paciente B) y un mínimo de un $8,5 \%$ (paciente G). (Figuras 2, 3 y 4 ). 

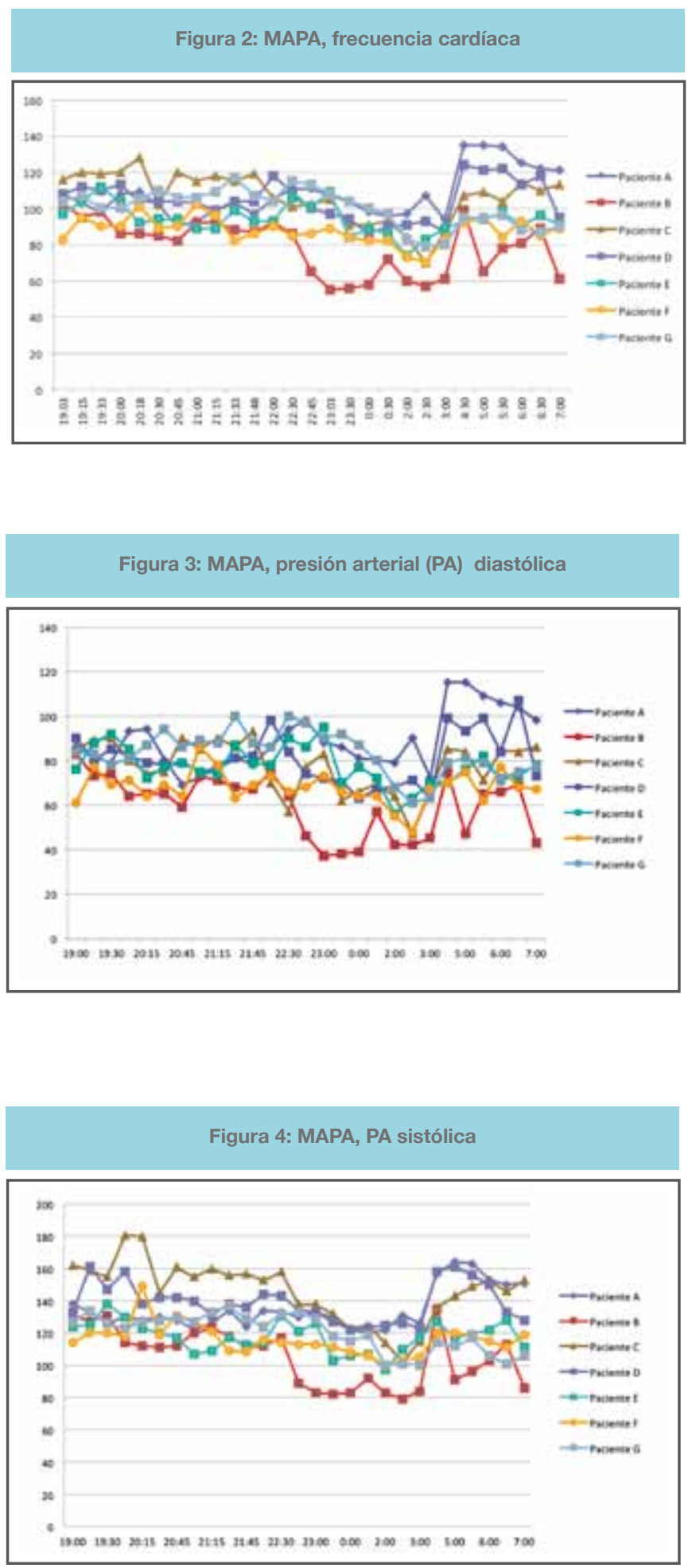

\section{Discusión}

En este estudio se confirmó un aumento estadísticamente significativo del diagnóstico electrocardiográfico de lesiones subepicárdicas en relación al estrés producido por el terremoto del 27 de febrero de 2010.

Esto coincidió con un aumento de frecuencia cardíaca y presión arterial, especialmente diastólica en el registro de los pacientes con MAPA al momento del terremoto, fenómeno ya observado durante el terremoto de Taiwán de 1999, donde se documentó en 15 pacientes con monitoreo de Holter un incremento súbito de frecuencia cardíaca con un cambio en su variabilidad que sugiere un predominio del sistema simpático durante el episodio de estrés ${ }^{8}$, fenómeno no documentado en aquellos pacientes en tratamiento con B-bloqueadores ${ }^{9}$.

De los pacientes con ECG compatibles con lesión subepicárdica durante el periodo post terremoto, un tercio no tenían antecedentes mórbidos.

Cabe destacar que se invirtió la relación porcentual de imagen electrocardiográfica de lesión subepicárdica entre hombres y mujeres, con un incremento marcado del diagnóstico en mujeres, situación que podría sugerir episodios de miocardiopatía de estrés como fue observado post terremoto de Nigata en Japón el año $2004^{10}$. Sin embargo, en nuestro trabajo no fue posible confirmar esta situación dado el bajo número de pacientes en que fue posible documentar el estudio coronariográfico (33\%). A pesar de esto, en aquellos pacientes en los que se pudo obtener datos, sólo un paciente tenía coronariografía normal.

Dentro de las debilidades de este estudio cabe mencionar que no fue posible rescatar los antecedentes de la totalidad de los pacientes en los que se diagnosticó lesión subepicárdica en el período post-terremoto. Por otra parte, el aumento del diagnóstico electrocardiográfico de lesiones subepicárdicas muy probablemente está subestimado, ya que a pese a que el servicio de Telemedicina siempre estuvo operativo, hubo interrupción de comunicaciones desde las zonas más afectadas. La totalidad de exámenes con diagnóstico de lesión subepicárdicas se recibieron desde la región Metropolitana, V y VI regiones en circunstancia que el epicentro fue en la VIII región.

Cabe destacar que este es el primer estudio realizado con Telemedicina en estas circunstancias permitiendo un registro en tiempo real a lo largo del país. 


\section{Referencias:}

1. OGAWA K; TSUJI I; SHIONO K; HISAMICHI S. Increased acute myocardial infarction mortality following the 1995 great Hanshin-Awaji earthquake in Japan. Int J Epidemiol. 2000; 29:449-455.

2. CHING-HONG T; FOR-WEY L; SHING-YAW W. The 1999 Ji-Ji (Taiwan) Earthquake as a Trigger for Acute Myocardial Infarction. Psychosomatics. 2004; 45:477-482

3. KLONER, L Natural disaster plus wake-up time: a deadly combination of triggers. Am Heart J. 1999; 137: 779-781

4. KARIO K; MC EWEN B; PICKERING G. Disasters and the Heart: a Review of the Effects of Earthquake-Induced Stress on Cardiovascular Disease. Hypertens Res. 2003; 26: 355-367.

5. WALCZEWSKAJ; RUTKOWSKIK; WIZNER B; CWYNAR M; GRODZICKI T. Stiffness of large arteries and cardiovascu- lar risk in patients with post-traumatic stress disorder. European Heart J. 2011; 32: 730-736.

6. TOMLINSON L; COCKCROFT J. Post-traumatic stress disorder: breaking hearts. European Heart J. 2011; 32: 668-669.

7. DIMSDALE J. Psychological Stress and Cardiovascular Disease. J Am Coll Cardiol. 2008; 51:1237-1246.

8. LIN LY; WU CC; LIU YB; HO YL; LIAU CS; LEE YT. Derangement of heart rate variability during a catastrophic earthquake: a possible mechanism for increased heart attacks. Pacing and clinical electrophysiology. 2001; 24: 1596-1601.

9. HUANG JL; CHIOU CW; TING CT; CHEN YT; CHEN SA Sudden changes in heart rate variability during the 1999 Taiwan earthquake. Am J Cardiol. 2001; 87: 245-248.

10. WATANABE, KODAMA,AIZAWA. Impact of Earthquakes on Takotsubo Cardiomyopathy. JAMA. 2005; 305:306. 